Running Head: CONTACT WITH EX AND PSYCHOLOGICAL DISTRESS

\title{
Contact with an Ex-partner is Associated with Psychological Distress after Marital Separation
}

Karey L. O’Hara, ${ }^{1}$ Austin M. Grinberg, ${ }^{2,3}$ Allison M. Tackman, ${ }^{4}$ Matthias R. Mehl, ${ }^{4}$ \& David A.

\author{
Sbarra $^{4}$ \\ ${ }^{1}$ REACH Institute, Arizona State University \\ ${ }^{2}$ VA Greater Los Angeles Healthcare System \\ ${ }^{3}$ David Geffen School of Medicine at University of California, Los Angeles \\ ${ }^{4}$ Department of Psychology, University of Arizona
}

In Press, Clinical Psychological Science

Final Accepted Version, 10/24/2019

Copyright held by SAGE Publications, Inc. This paper is not the copy of record and may differ slightly from the final published version. Please do not copy or cite without authors' permission.

Acknowledgements: This research was supported by a grant from the National Institute of Child Health and Human Development (R01HD069498). The first author's work on this paper was supported by a postdoctoral fellowship provided by the National Institute on Drug Abuse (T32DA039772) through the Psychology Department and the Research and Education Advancing Children's Health (REACH) Institute at Arizona State University. 


\section{Author Contributions}

KLO, AMG, and DAS developed the paper concept. KLO and AMG performed the analyses with input from DAS. KLO, AMG, and DAS drafted the manuscript. MRM and AMT provided critical revisions. AMT and MRM organized and provided critical oversight of all EAR data collection and coding. DAS and MRM designed the study. All authors approved the final version of the manuscript for submission.

\section{Declaration of Conflicting Interests}

The authors declare no conflicts of interest with respect to their authorship or the publication of this article.

\section{Open Science Practices}

A preregistration of the analytic plan as well as all data and materials used for the present report are publicly available via the Open Science Framework and can be accessed at https://osf.io/93yrk/.

\section{Correspondence}

Correspondence should be addressed to Karey L. O'Hara, REACH Institute, Department of Psychology, Arizona State University, 900 S McAllister Ave., Tempe, AZ 85287, USA. Phone: 520-271-9830. Email: klohara@asu.edu. 


\begin{abstract}
This study examined the association between naturalistically-observed in-person contact with one's ex-partner and separation-related psychological distress (SRPD). 122 recentlyseparated adults were assessed using the Electronically Activated Recorder (Mehl, 2017) on three occasions across five months. The association between in-person contact with one's expartner, as a between-person variable, and concurrent SRPD was not reliably different from zero, nor was the time-varying effect of in-person contact. However, more frequent in-person contact with one's ex-partner predicted higher SRPD two months later, above and beyond the variance accounted for by concurrent in-person contact, demographic, relationship, and attachment factors. Follow-up analyses yielded that this effect was only present for people without children; a one standard deviation increase in in-person contact offset and slowed the predicted decline in SRPD over two months by $112 \%$. Our discussion emphasizes new ways to think about the role of interpersonal contact in shaping adults' psychological adjustment to separation over time.
\end{abstract}


Contact with an Ex-partner is Associated with Psychological Distress after Marital Separation Marital separation and divorce are associated with risk for a range of negative outcomes, including clinically-significant mood disturbance, suicidal ideation, and suicide attempts (Sbarra, Emery, Beam, \& Ocker, 2014; Weissman et al., 1999). For anyone who has struggled with a breakup or marital separation, questions about whether, how, and how often to be in touch with an ex-partner have enormous importance. Following divorce, $55 \%$ of adults report having monthly, and 32\% report having weekly, contact with their ex-partner (Masheter, 1997). Similarly, a large, nationally representative study of ever-divorced men and women in the Netherlands found that roughly $50 \%$ of adults report continued contact with their ex-spouse up to 10 years following relationship termination (Fischer, de Graaf, \& Kalmijn, 2005). These high rates of ongoing contact between ex-partners raise several basic yet important empirical questions: Does contact with an ex-partner interfere with psychological recovery from separation in a discernable way? Does contact with an ex-partner increase separation-related emotional distress? Are some people, perhaps those with certain attachment orientations, particularly affected by ongoing interactions with an ex-partner?

Early studies on this topic indicated that contact with an ex-partner is associated with worse outcomes post-divorce (Brown, Felton, Whiteman, \& Manela, 1980). Likewise, a large prospective study of young adults in romantic relationships found that higher frequency of contact following a breakup was associated with declines in life satisfaction (Rhoades, Kamp Dush, Atkins, Stanley, \& Markman, 2011). To make the story more complicated, a study of young adults who recently experienced a romantic breakup reported higher levels of both love and sadness on days that they were in contact with their ex-partner (Sbarra \& Emery, 2005b). In a more recent study following non-marital romantic relationship termination, non-sexual contact 
with an ex-partner was associated with poorer adjustment, but only in adults reporting lower levels of acceptance (Mason, Sbarra, Bryan, \& Lee, 2012). Other studies find no association between ongoing contact with an ex-partner and psychological outcomes (Masheter, 1997).

\section{Toward an Objective Assessment of Contact with an Ex-Partner}

Most studies investigating this association rely on self-report data, which, through their dependence on reflected subjective representations of the experiences surrounding a separation, might accentuate or bias estimates of contact with an ex-partner (Mehl, Robbins, \& Deters, 2012; Vazire \& Mehl, 2008). For example, it is possible that the high personal relevance that seeing an ex-partner often has might lead to overestimations of how much time was spent together. Alternatively, the potentially high emotional toll that can accompany meeting with an ex-partner might also lead to retrospectively downplaying the amount of contact. In this regard, the Electronically Activated Recorder (EAR; Mehl, 2017; Mehl, Pennebaker, Crow, Dabbs, \& Price, 2001) can provide a psychometrically complimentary, "objective" (in the sense of "traceable"), and ecologically-valid way of measuring ongoing in-person contact with an ex-partner.

The EAR is an ecological momentary assessment tool that has been thoroughly validated for the study of social phenomena as they unfold in people's daily lives. Researchers have used the EAR to study a wide range of intra and interpersonal processes, including how families cope with stress (Slatcher \& Robles, 2012; Tobin et al., 2015) and trauma (Alisic, Barrett, Bowles, Conroy, \& Mehl, 2016), the consequences of swearing on emotional support and depression symptoms for health-compromised women (Robbins et al., 2011), and the role of social integration on divorcing adults' emotional and physical health (Hasselmo et al., 2018).

\section{The Person and the Context: The Moderating Role of Individual Differences}


Another possible explanation of the mixed results found in the contact-distress literature is a dearth of studies investigating moderators. Psychological outcomes following ongoing contact with an ex-partner are likely moderated by a range of variables, including relationship orientations (e.g., attachment insecurity), individual psychological processes such as a history of major depressive disorder and a tendency toward maladaptive repetitive thinking (Davis, Shaver, \& Vernon, 2003; Lee, Sbarra, Mason, \& Law, 2011; Sbarra et al., 2014), as well as ongoing attachment to a former partner (see Borelli, Sbarra, \& Mehl, 2019). Illustratively, a study investigating the effects of ongoing contact with an ex-partner following marital separation found that the association between contact and psychological adjustment was moderated by the degree to which individuals had accepted their separation, such that adults who remained in contact with their ex-partner and were less accepting of the separation exhibited poorer adjustment as compared to adults with higher levels of separation acceptance (Mason et al., 2012). The continued search for substantive moderators is a key direction for research in this area (Mason \& Sbarra, 2012).

\section{The Current Study}

The current study examined change in self-reported separation-related psychological distress (SRPD) as a function of naturalistically-observed (measured via the EAR) in-person contact (i.e., physically in the same space and interacting in some capacity; does not include phone calls or social media contact) with one's ex-partner in a sample of 122 recently divorced/separated adults assessed three times over five months. This work is the first to examine the ways in which naturalistically-observed ongoing in-person contact with an expartner may be associated with adults' psychological responses to separation. As part of our preregistered analytic plan (https://osf.io/w4xvq/), we predicted a positive within-occasion 
association between the amount of (EAR-observed) in-person contact participants had with their ex-partner and their SRPD. We expected that this effect would weaken over time. In the preregistered plan, we also hypothesized that the association between observed in-person contact with one's ex-partner and SRPD would be stronger for people high on attachment anxiety and lingering attachment to their ex-partner. Finally, in a series of exploratory analyses, we tested whether the effect of in-person contact on SRPD persisted over time.

\section{Method}

\section{Participants}

The data reported in the current paper come from a larger study of 140 communitydwelling adults following a recent marital separation or divorce (see Borelli et al., 2019; Hasselmo et al., 2018; Manvelian, Bourassa, Lawrence, Mehl, \& Sbarra, 2018; Milek et al., 2018; Tackman et al., 2019). An Institutional Review Board approved all study protocols prior to data collection. The analyses reported here include all cases that had adequate EAR data (i.e., at

least 30 valid sound files) for at least one study occasion. The current sample is comprised of 122 adults (35 men, 87 women) between age 24-65 $(M=43.84, S D=10.52)$. We used an intent-to-treat approach for longitudinal analyses. Power analyses conducted through Monte Carlo simulation in Mplus prior to grant submission indicated that the target sample size of 120 participants would yield between .78 and 1.0 power to detect small main or moderating effects $(d=.10$ to .20$)$ in linear growth models of psychological distress (assuming a standardized linear change of $d=.23$ per 3-month assessment period).

Participants were married for an average of 13.4 years $(S D=9.08$ years $)$ before separation, and entered the study, on average, within 3.8 months $(S D=2.17)$ of their physical separation from their spouse. Ninety-nine percent of the sample identified as being physically 
separated from their spouse, $19 \%$ as legally divorced, $23 \%$ as legally separated, and $57 \%$ as physically separated but with no legal actions filed. The sample is comprised of participants who identify as Non-Hispanic White (62.3\%), Hispanic (22.1\%,) African American (4.9\%), Asian (2.5\%), and Native American (1.6\%), with 5.7\% who identified their ethnicity in the "Other" category. Participants reported a wide income range with roughly $23.8 \%$ making less than $\$ 11,999$ per year, 32.8\% making between $\$ 12,000$ and $\$ 34,999,29.5 \%$ making between $\$ 35,000$ and $\$ 74,999,7.4 \%$ making more than $\$ 75,000$ per year. The remaining $5.8 \%$ either declined to answer or indicated that they did not know their annual income. Relative to participants who did not provide sufficient valid EAR data, those participants included in the current subsample had, on average, longer marriages $\left(\mathrm{M}_{\text {subsample }}=13.4\right.$ years; $\mathrm{M}_{\text {excluded }}=6.21$ years; $\mathrm{F}[1,125]=7.87, \mathrm{p}=$ .006). Participants did not differ significantly on any additional demographic variables, including sex, age, race, income, or having children $(p=.05-.96)$.

\section{Procedures}

Participants were recruited via the legal system (family and conciliation courts), online and newspaper advertisements, and divorce support groups in a large Southwest metropolitan area of the United States to enroll in a study investigating adults' adjustment following marital separation/divorce. Eligibility criteria included having been married for at least three years and cohabited for at least 2 years with their recently separated/divorced partner.

The study consisted of five waves of data collection over five months. In this paper, we use data from the $1^{\text {st }}, 3^{\text {rd }}$, and $5^{\text {th }}$ study visits, which were completed in-home or in the lab, according to participants' preferences. These assessments coincided with the weekend periods (i.e., beginning Friday until they went to bed on Sunday) during which participants wore the Electronically Activated Recorder (EAR; Mehl et al., 2012). It should be noted that participants 
completed self-reported measures during the week prior to the 3-day EAR assessment period. Assessment measures (subjective and objective) are described as concurrent throughout the paper if completed within the same time point $\left(1^{\text {st }}, 3^{\text {rd }}\right.$, or $5^{\text {th }}$ study visit).

In-person contact with one's ex-partner was assessed by the EAR method. Please see https://osf.io/93yrk/ to access the full set of study procedures and measure and https://psyarxiv.com/mgzcy/ for a paper describing the coding process and best practices in using the EAR. The EAR records sound bites on a customizable time scale. In tracking moment-tomoment ambient sounds, the EAR yields a log of individuals' social interactions as they naturally unfold. In the current study, participants' behavioral data were captured in 30-second clips every 12 minutes during waking hours over the course of one weekend (with a 6-hour overnight blackout; i.e., pre-set non-recording, period that was calibrated to participants' reported habitual weekend sleep times). The time scale was determined in part, based on the "thin-slice" literature indicating that 30 seconds of ambient sounds provide surprisingly much information (e.g., Ambady, LaPlante, \& Johnson, 2001). After completion of the study, trained observers coded the sound files using a standardized coding system (available at https://osf.io/4yb97/). The coding procedures include extensive use of the context of preceding and following sound files to enhance their accuracy. For example, if the ex-partner was audible in the first sound file, not audible 12 minutes later in the second sound file, but then again in the third sound file (and, say, the context had not changed, the participant was still at a cafe), then the coder would use this contextual information to make a determination (i.e., code the expartner as present in the second sound file). Importantly, as coders listen to more and more sound files by a participant, their information about the participant's social network increases and, over time, through voices that appear and reappear, through conversations that happen when certain 
voices are present, through voices that are only there in certain contexts, and through paraverbal features (e.g., voice inflection), coders tend to develop a good grasp of the basic "who is who." And, as they learn something new about the participant, they often go back and adjust their initial coding.

\section{Measures}

Separation-related psychological distress (SRPD). SRPD was measured by four selfreport questionnaires: Beck Depression Inventory (BDI), Impact of Events Scale-Revised (IESR), Loss-of-Self/Rediscovery-of-Self (LOSROS), and Inventory of Complicated Grief (ICG). The BDI (Beck, Steer, \& Carbin, 1988) was included to assess depressive symptoms, as they have been linked to post-relationship dissolution adjustment in previous studies (e.g., Sbarra, Boals, Mason, Larson, \& Mehl, 2013; Sbarra \& Emery, 2005a). The IES-R (Weiss, 2007) was used to assess emotional distress (i.e., intrusive thoughts and somatic hyperarousal) related to the relationship dissolution. It has been used in previous studies investigating emotional adjustment to a range of stressful life events, including relationship dissolution (e.g., Chung et al., 2003; Sbarra \& Emery, 2005b). The LOSROS is a measure a perceived loss and rediscovery of the self after relationship dissolution that has been linked to emotional recovery after divorce (Lewandowski \& Bizzoco, 2007). The ICG (Prigerson et al., 1995) measures the degree of grief associated with the loss of the romantic relationship, and has been frequently used as an indication of post-relationship dissolution adjustment (e.g., del Palacio-González, Clark, \& O’Sullivan, 2017; Verhallen, Renken, Marsman, \& Horst, 2019).

The measures were rescaled using linear transformations to Percentage of Maximum Possible (POMP) scores on a scale of 0 to 100 (Cohen, Cohen, Aiken, \& West, 1999), and then combined into a standardized mean composite of SRPD. This composite has been used in 
previous studies of divorced and separated adults (e.g., Bourassa, Tackman, Mehl, \& Sbarra, 2019). For this study, the focal time-varying repeated measures dependent variable was score on SRPD on 3 occasions (Month 1 mean $=30.42$ points; Month 3 mean $=24.15$ points; Month 5 mean $=20.69)$. The reliability of change in SRPD was calculated according to the application of the generalizability theory method outlined in Bolger and Laurenceau, 2013. Based on item scores comprised of each of the four scales across the three assessment occasions, the reliability of change index was .77 , indicating that the degree of reliability in within-person differences in change over time was moderate (Shrout, 1998).

In-person contact with an ex-partner. EAR data accumulated to approximately 90 sound bites or 45 minutes of recording per day (two full days, Saturday and Sunday, and some hours on Friday night, per measurement occasion). For each sound file, the coders identified whether the participant was or was not with their ex-partner (i.e. physically in the same space and interacting in some way). The coders further coded whether, in a given sound file, the participant was sleeping or was deemed not wearing the EAR device. These binary codes of being with the ex-partner in all compliant and waking sound files were then aggregated within visit to yield an estimate of the proportion of time participants spent engaged in in-person contact with their ex-partner within measurement occasion (i.e. within a weekend). Scores on EARobserved in-person contact with the participant's ex-partner (WithEx) were derived from EAR data across three measurement occasions (1-month, 3-months, 5-months; ICCs for intercoder reliability were $.823, .946$, and .648 , respectively) and were considered a time-varying predictor. We multiplied proportion scores by 100 for easier interpretation.

Attachment anxiety. The 6-item anxiety subscale and 6-item avoidance subscale of the short version of the Experiences in Close Relationships Scale (ECR; Wei, Russell, Mallinckrodt, 
\& Vogel, 2007) assessed attachment style on a 7-point scale $(1=$ strongly disagree to $7=$ strongly agree) with higher scores indicating greater attachment anxiety or avoidance. The subscale items in the current sample demonstrated adequate scale reliability $\left(\alpha_{\text {anxiety }}=0.77\right.$, $\alpha_{\text {avoidance }}=0.72$ ).

Lingering attachment. Participants reported their perceptions of attachment to participants' former partner using a modified version of the WHO-TO scale (Fraley \& Davis, 1997; Hazan \& Zeifman, 1994). The WHO-TO scale is comprised of 16 items rated on a 7-point scale $(1=$ strongly disagree to $7=$ strongly agree $)$ with higher scores indicated greater attachment to participants' former partner. The scale assesses a number of attachment features and functions within the context of a recent separation/divorce by asking participants to rate the extent to which they remained attached to their former partner as reflected in their experience of separation distress, the degree to which they desire to use their ex-partner as a secure and safe base, and how much they wish to maintain proximity. Illustrative items include "I miss my expartner when she/he is away" and "My ex-partner is the person I want to talk to when I'm worried about something." In the current study, the self-reported lingering attachment items demonstrated strong scale reliability $(\alpha=0.94)$.

Demographic and relationship covariates. Beyond the competing predictors of attachment orientations and lingering attachment to an ex-partner, we included two sets of a priori and preregistered covariates in the analyses based on previous research studies implicating these variables as possible predictors of post-divorce adjustment (Mason, Law, Bryan, Portley, \& Sbarra, 2012; Sbarra \& Emery, 2005a; Wang \& Amato, 2000). Demographic covariates included age and sex. Relationship-specific covariates included time since separation (in months), length 
of relationship (in months), and perceived responsibility for the separation/divorce on a 4-point Likert scale ( 1 = participant was totally responsible, 4 = partner was totally responsible).

\section{Data Analysis}

Analyses were conducted in a multilevel modeling framework using the nmle package in R (Pinheiro, Bates, DebRoy, \& Sarkar, 2019). Full information maximum likelihood estimation (FIML) handled missing data $(<1 \%$ on SRPD dependent variable; $7.1 \%$ on the WithEx predictor variable). We observed positive skew and kurtosis in the distribution of WithEx raw scores, but chose not to transform this variable based on our desire to maintain an interpretable scale and to avoid documented cautions against data transformations including unstable and out-of-range parameter estimates (Feng et al., 2014; O’Hara \& Kotze, 2010). ${ }^{1}$ We first tested a baseline model to examine the functional form of change in SRPD scores across the study period. We then added disaggregated (person specific-mean variable represents a Level-2 [L2] between-person effect and person mean-centered variable represents a Level-1 [L1] within-person effect; Curran, Lee, Howard, Lane, \& MacCallum, 2012) fixed and random effects of WithEx to the model (Table 2, Model 1). Next, we included our prespecified covariates, including age, sex, length of separation, relationship length, and perceived responsibility for the separation to examine how the trajectory of SRPD predicted by WithEx changed after accounting for relevant covariates (Table 2, Model 2). Finally, we tested three moderation models to assess our preregistered hypotheses that the association between in-person contact with the ex-partner and SRPD would be conditioned by time (described in-text), anxious attachment style (Table 2, Model 3), and lingering attachment to the ex-partner (Table 2, Model 4). We added an exploratory analysis to

\footnotetext{
${ }^{1}$ We also specified all models with a log-transformed (log base 10) WithEx variable, after adding a constant of .001, and found no substantial differences in our conclusions. Details of these analyses are provided in Supplementary Materials.
} 
examine the lagged effect of in-person contact on SRPD 2 months later (Table 3, Model 5 and Model 6).

In all models, we first attempted model convergence with all L1 random effects (time and WE_within) and the L2 effect of interest (WE_between). If the model failed to converge, we retained all L1 random effects only. In instances where the model would still not converge, we removed the random effect of time in an effort to adequately model the primary within-person effect of interest (WE_within). All code of statistical modeling for the current study is available via Rmarkdown posted at https://osf.io/w4xvq/.

\section{Results}

Table 1 displays descriptive statistics and intercorrelations of all study variables. From the perspective of the group average, it was rare for the participants to be with an ex-partner during EAR data collection; the within-occasion group means ranged between 1-2\% of the weekend data where the presence of the ex-partner was detected. It is important to note though, that the variability was high; some participants spent as much as $36 \%$ of a weekend with their ex-partner. The grouplevel average is low in part due to a large number of participants spending no time at all with their ex-partner.

\section{Analysis of Preregistered Hypotheses}

Our model building approach called first for specifying the nature of change in SRPD over the 5-month study period. SRPD scores declined linearly over the study period, $b=-4.80$, $S E=0.55,95 \% \mathrm{CI}=-5.88,-3.73, p=<.001$. For our main analyses, neither the between-person (grand mean-centered) effect of WithEx, $(b=46.32, S E=25.99,95 \% \mathrm{CI}=-4.84,97.47, p=$ $.077)$, nor the within-person (person mean-centered) effect of WithEx, $(b=-31.72, S E=19.89$, $95 \% \mathrm{CI}=-70.70,7.26, p=.112)$, was associated with concurrent SRPD in a manner that reliably 
differed from zero. The between-person effect was in the hypothesized direction with greater average-level in-person contact with one's ex-partner being associated with higher levels of SRPD across the study period (Table 2; Model 1). Model 2 illustrates the full multilevel model; older participants reported higher levels of SRPD on average, as compared to younger participants $(b=0.28, S E=0.13,95 \% \mathrm{CI}=0.03,0.54, p=.035)$. The extent to which the participant perceived that their ex-partner was responsible for the breakup $(b=3.53, S E=1.18$, $95 \% \mathrm{CI}=1.23,5.83, p=.004)$ predicted higher levels of SRPD. Further, there were main effects of both attachment anxiety $(b=3.99, S E=0.94,95 \% \mathrm{CI}=2.16,5.82, p<.001)$ and attachment avoidance $(b=2.66, S E=1.08,95 \% \mathrm{CI}=0.56,4.76, p=.014)$. We found no support for the hypothesis that the effect of in-person contact with the ex-partner is moderated by time (i.e. a weakening of the effect over time), nor that the in-person contact effect is significantly moderated by attachment anxiety (Table 2; Model 3). We observed a significant interaction between the within-person effect of in-person contact with the ex-partner and lingering attachment on SRPD (Table 2, Model 4), but the effect was marginal after accounting for covariates $(p=.044)$ so we are not confident that it is a reliable effect. Overall, our preregistered analyses did not support the hypothesis that in-person contact with an ex-partner is associated with worse concurrent SRPD, nor does the effect seem to be moderated by time since separation or attachment-related individual differences.

\section{Exploratory Analyses}

Upon examining the zero-order correlations between study variables at each occasion, we observed strong prospective correlations between WithEx and SRPD. We formally tested whether this potential lagged effect was evident in the full multilevel change model above-andbeyond concurrent in-person contact with one's partner. Results of this analysis are reported in 
Table 3 (Model 5). After accounting for the systematic rate of decline in SRPD over time, concurrent in-person contact relative to one's own mean, and overall average in-person contact, the lag-1 effect of in-person contact with one's ex-partner was reliably different than zero $(b=$ $123.15, S E=32.32,95 \% \mathrm{CI}=59.74,186.57, p=<.001)$; greater in-person contact with one's expartner (relative to one's own mean) predicted higher levels of SRPD two months later. We found no support for the hypothesis that the lagged effect of in-person contact with the expartner is moderated by lingering attachment or attachment anxiety $(p=.183$ and .900 , respectively). However, we did find that the lagged effect of in-person contact with the expartner is moderated by whether or not the participant has children (biological or stepchildren) with their ex-partner $(b=-123.93, \mathrm{SE}=71.60,95 \% \mathrm{CI}=-353.71,-74.15, \mathrm{p}=.004)$. Model 6 (Table 3) illustrates the results of the full, covariate-adjusted multilevel model. As shown, the interactive lagged effect remained reliably different from zero, $b=-233.41, S E=74.54,95 \% \mathrm{CI}$ $=-356.53,-81.29, p=.029$, when accounting for concurrent in-person contact, as well as the demographic, relationship-specific, and attachment covariates. Simple slopes analyses indicated that for those without children, the relation between lagged contact with the ex-partner and SRPD was positive and significant $(p<.001)$ while for those with children, the relation was not significant $(p=.953)$; see Figure 1 for an illustration of the interaction. Within this model, for participants without children, a one standard deviation increase in in-person contact with one's ex-partner (relative to one's own mean) is associated with a 0.28 unit increase in SRPD two months later. This effect is roughly equivalent to $112 \%$ of the magnitude of change in SRPD observed across each of the three visits. Said differently, a one standard deviation increase in inperson contact with one's ex-partner offsets and slows the amount of decline people experience in SRPD over two months by this amount, and this effect operates independently of 
demographics, relationship characteristics, or attachment orientations, all of which are reliable predictors of separation-related adjustment. Figure 2 illustrates the relative magnitude of change in SRPD associated with a 1 SD increase in each of the five significant predictors in this model. In contrast to the negative effect associated with the passage of time, prior in-person contact with one's ex-partner, as well as attachment style and perceived responsibility for the breakup, are associated with an increase in SRPD over time.

We also speculated that the observed lagged effect of WithEx may be conditioned by two composites of subjective emotions (positive and negative) participants expressed with regard to their ex-partner. An illustrative item is "When you think about your ex-partner, to what extent do you experience hate?" We found no interaction effects of WithEx and positive emotions or negative emotions. We also attempted to investigate whether the affective quality of the interaction (i.e., proportion of contact that was characterized by expressions of anger) changed the nature of the effect. However, the size of the relevant subsample (only those who had any EAR-observed contact with their ex-partner during the study period) was too small to draw any meaningful conclusions.

\section{Discussion}

The current study examined the association between naturalistically-assessed in-person contact with an ex-partner and SRPD in a sample of 122 recently separated adults. Contrary to the hypothesis in our preregistered analysis plan, in-person contact with one's ex-partner was not a significant predictor of concurrent SRPD. Moreover, we found no evidence that theoreticallydriven moderators, including lingering attachment to one's ex-partner or attachment orientations, moderated the association between in-person contact and SRPD. However, in a series of exploratory analyses, we found that for some individuals, the lagged effect of in-person contact 
with one's ex-partner - that is, in-person contact that was objectively measured two months prior - was associated with current SRPD, and this effect operated above-and-beyond the passage of time as well as the concurrent effect of in-person contact on SRPD and relevant covariates. Through the repeated assessment of in-person contact with an ex-partner, we were able to decompose this variable into its between- and within-person variance components, and we found it is the within-person variable that is predictive of future distress, not people's general tendency to be in in-person contact with their ex-partner (relative to others in the sample). Moreover, this effect was moderated by whether or not the couple had shared children (biological or step-children). For those adults without shared children, contact with their expartner two months prior was associated with worse SRPD; for adults with shared children, the association between contact and distress was not reliably different from zero.

What might explain the absence of a concurrent effect but the presence of a lagged effect? To the extent that the prospective effect proves replicable in future studies, it may be that although prior cross-sectional research suggests a concurrent association, in fact, longitudinal studies are better suited for capturing the time-based emergence of the association. Theoretically, it is plausible that in-person contact with one's ex-partner is not associated with overtly negative effects at the time, but rather sets in motion a process that is ultimately associated with worsened distress and slowed psychological adjustment. For example, it may be that contact arouses multiple emotions simultaneously which, when assessed as a global indicator of distress, is unlikely to be predictive in either direction. The current data cannot speak to the causal or mechanistic processes underlying the observed lagged effect, but in future research, it will be crucial to study the potential time-based mediational processes that explain it. 
Why would the relative level of contact, but not the overall level of contact with an expartner, be associated with SRPD for individuals without children? A within-person effect in the absence of a between-person effect is conceptually intriguing because it highlights the importance of context as it pertains to how psychological adjustment is related to post-separation behaviors. Perhaps it is not the case that contact with an ex-partner is inherently adaptive or maladaptive for any given person. Rather, it may depend on the predictability and ordinariness of these interactions. People tend to habituate to normal or expected levels of any experience and departures from that set-point are what is noticed and hedonically important (Fujita \& Diener, 2005). For example, if your custody arrangement involves seeing your ex-partner briefly two times per week (e.g., to exchange children), you are likely to become accustomed to this as a part of your normal life. Thus, as long as the interactions proceed as expected, they may not have strong emotional implications. On the other hand, unexpected or inconsistent interactions may spark stronger reactions (e.g., an ex-partner showing up unannounced at a mutual friend's event; the need for an ad-hoc meeting to resolve a financial issue).

We also expected that the main effect of ongoing in-person contact on SRPD would be moderated by well-documented interpersonal factors tied to increased risk, such as degree of lingering attachment to the ex-partner and global relationship orientations. In particular, we suspected that individuals who report a high degree of continued emotional reliance on their expartner and/or an attachment style characterized by high anxiety or avoidance, would be the most negatively affected by continued in-person contact with the ex-partner. Contrary to our hypothesis, we found that these factors exerted main effects on SRPD but there was no evidence of moderating effects associated with attachment orientations. This finding partially contradicts a previous study by Mason and colleagues (2012) that found that lingering attachment to an ex- 
partner (or lower acceptance of the separation) moderated the association between in-person contact and psychological adjustment (operationalized as separation-related emotional intrusion, hyperarousal, and avoidance behaviors). It is possible that methodological differences between the studies explain the differences in findings (the use of the EAR vs. self-reported contact). It is also important to consider that the specific sampling scheme for the EAR used in this study (i.e., weekend days only) may affect the strength of observed effects.

Our observation that the lagged effect of in-person contact with one's ex-partner on concurrent SRPD was conditioned by whether or not the participant had children with their expartner was not unexpected, but the direction of the effect (significant only for adults without shared children) was somewhat surprising. However, differential effects of maintaining contact for adults with and without shared children does align, in-part, with a previous study that found adults with shared children have both more friendly and more antagonistic contact following marital dissolution as compared to divorced adults without children (Fischer et al., 2005). One potential explanation for this difference is that contact with an ex-partner may take on a qualitatively different form for those who have children together. For example, much of the contact between ex-partners with children may be routine and business-like (e.g., dropping off and picking up children), whereas contact between ex-partners who do not have a necessary reason to meet is likely to be more emotionally-laden and attributions about the contact may be more centered around the separation (e.g., recapitulating the reasons, pondering whether or not it was the right decision). Further, the finding that the effect of contact with an ex-partner depends on how it compares to one's own average (and not the overall between-person level of contact) may have different implications depending on whether or not there are children involved. It may be that for those with children, because they are more likely to have a set routine than those 
without children, the average effect may be zero because both more (e.g., contact outside of the childcare arrangement) and less contact (e.g., not showing up for a planned exchange) than usual has implications for distress. Contextualizing these findings in an empirical way is an important future research objective, particularly given the fact that the rates of divorce are higher in couples without children (Bramlett \& Mosher, 2002).

The implications of the current study span theoretical, methodological, and applied domains. First, the takeaway message is that maintaining in-person contact with an ex-partner can impede the "natural" recovery process tied to the passage of time for individuals without children, and that these effects may not be experienced immediately. Although the mechanism is not yet well articulated, and likely idiographic (i.e., for some it may be because it precludes the emotional and physical space needed for the development of new relationships, whereas for others it may actively maintain emotional connection within the old relationship), ongoing inperson contact appears to keep some people stagnant — or, at least, is associated with less improvement over time - and interferes with one's ability to move on. Second, the methodological innovations of the current study illustrate the need to study these complex interpersonal and intrapersonal processes affecting adjustment after separation and divorce using intensive, longitudinal, and multimethod assessment procedures. An important future direction will be to extend the use of this methodology using a more comprehensive sampling scheme (e.g., longer monitoring period including both weekday and weekend sampling; higher frequency sampling).

Finally, establishing in-person contact with an ex-partner as a behavioral predictor of psychological distress following divorce and separation may have important implications for psychosocial and behavioral interventions to reduce risk. Given its behavioral nature, 
maintenance of in-person contact may be more amenable to change than other risk factors, including attachment orientations. At the same time, it is crucial to recognize that many adults do not have the freedom to simply cease in-person contact with their ex-partner. People stay in contact with their ex-partner for diverse and valid reasons beyond co-parenting arrangements, including financial needs, shared social networks, or joint business investments. Thus, research in this area should continue to develop an understanding of the nuances that condition and explain this finding. For example, are there characteristics of the contact (for an example see Masheter, 1997), such as affective quality or degree of hostile conflict, that drive this effect? Is it any contact at all (i.e., via phone, text, email, social media), or only in-person contact? The answers to these questions will not only develop a richer understanding of the phenomenon and contribute to theory development, but also provide important insights into how one might manage contact with an ex-partner under circumstances in which discontinuing contact is not possible. A key direction for future research is to recruit a large sample of adults with adequate variance in the degree of ongoing contact to further investigate how and for whom contact predicts psychological distress.

The results of this report should be considered in light of several limitations. Foremost, the sample size is relatively small, and the findings need to be replicated in a larger sample of recently divorced or separated adults. Second, although we preregistered our hypotheses and data analytic plan for the current study prior to data analysis, it was not proposed prior to data collection. Third, the order of assessments within each time point may have affected the nature of the observed findings. Future studies should consider counterbalancing the order of assessments (subjective vs. objective) to account for possible order effects. Similarly, the sampling scheme may not be optimal for capturing low base rate phenomena, such as in-person 
contact between ex-partners. Although the sampling rate is relatively high (i.e., five times per hour), for example compared to experience sampling, we only sampled over weekends. Thus, the results may be constrained by the lack of sampling during weekdays and it is possible that contact with an ex-partner is quantitatively and qualitatively different on weekends and weekdays. Finally, the study was observational in nature, and thus all reported associations are strictly correlational and preclude causal interpretations. Nevertheless, the current study serves as a foundation for future intervention studies that use an experimental design to manipulate contact between ex-partners to understand causal links between ongoing contact and SRPD.

To our knowledge, this is the first study to investigate within-person effects of in-person contact with an ex-partner on SRPD using a longitudinal and multimethod research design. The findings build on prior research and provide a clearer picture of these associations, without the bias of shared method variance and self-report of contact. The substantive conclusion suggests that for adults without shared children, more in-person contact with an ex-partner predicts higher SRPD two months later, after accounting for concurrent in-person contact, demographic, relationship, and attachment factors. More broadly, the findings highlight the importance of considering how the interpersonal process of contact between ex-partners shape intrapersonal adjustment over time in those experiencing marital dissolution. The chief contribution of this work is in showing that for some individuals, contact with an ex-partner is associated with greater emotional distress and slowed trajectories of psychological adaptation over time. This work, if replicated, has direct relevance for helping adults navigate the end of their marriage by elucidating factors that may contribute to risk for clinically-significant outcomes so that we can better develop targeted interventions aimed at preventing long-term negative effects of marital separation and divorce. 


\section{References}

Alisic, E., Barrett, A., Bowles, P., Conroy, R., \& Mehl, M. R. (2016). Topical review: Families coping with child trauma: a naturalistic observation methodology. Journal of Pediatric Psychology, 41(1), 117-127. https://doi.org/10.1093/jpepsy/jsv016

Ambady, N., LaPlante, D., \& Johnson, E. (2001). Thin-slice judgments as a measure of interpersonal sensitivity.

Beck, A. T., Steer, R. A., \& Carbin, M. G. (1988). Psychometric properties of the Beck Depression Inventory: Twenty-five years of evaluation. Clinical Psychology Review, $8(1), 77-100$.

Bolger, N., \& Laurenceau, J.-P. (2013). Psychometrics of intensive longitudinal measures of emotional states. In Intensive longitudinal methods: An introduction to diary and experience sampling research (pp. 127-141). Guilford Press.

Borelli, J. L., Sbarra, D. A., \& Mehl, M. (2019). Convergence of naïve coders and participant report ratings of attachment to a former partner: When we should be me. Journal of Social and Personal Relationships, 36(3), 977-999. https://doi.org/10.1177/0265407517749755

Bourassa, K. J., Tackman, A. M., Mehl, M. R., \& Sbarra, D. A. (2019). Psychological overinvolvement, emotional distress, and daily affect following marital dissolution. Collabra: Psychology, 5(1), 8. https://doi.org/10.1525/collabra.184

Bramlett, M. D., \& Mosher, W. D. (2002). Cohabitation, marriage, divorce, and remarriage in the United States (No. 23(22)). National Center for Health Statistics.

Brown, P., Felton, B. J., Whiteman, V., \& Manela, R. (1980). Attachment and distress following marital separation. Journal of Divorce, 3(4), 303-317. 
Chung, M. C., Farmer, S., Grant, K., Newton, R., Payne, S., Perry, M., ... Stone, N. (2003). Coping with post-traumatic stress symptoms following relationship dissolution. Stress and Health, 19(1), 27-36. https://doi.org/10.1002/smi.956

Cohen, P., Cohen, J., Aiken, L. S., \& West, S. G. (1999). The problem of units and the circumstance for POMP. Multivariate Behavioral Research, 34(3), 315-346. https://doi.org/10.1207/S15327906MBR3403_2

Curran, L., Lee, T., Howard, A., Lane, S., \& MacCallum, R. (2012). Disaggregating withinperson and between-person effects in multilevel and structural equation growth models. In J. Harring \& G. Hancock (Eds.), Advances in longitudinal methods in the social and behavioral sciences (pp. 217-253). United States of America: Information Age Publishing Inc.

Davis, D., Shaver, P. R., \& Vernon, M. L. (2003). Physical, emotional, and behavioral reactions to breaking up: The roles of gender, age, emotional involvement, and attachment style. Personality and Social Psychology Bulletin, 29(7), 871-884. https://doi.org/10.1177/0146167203029007006

del Palacio-González, A., Clark, D. A., \& O’Sullivan, L. F. (2017). Cognitive processing in the aftermath of relationship dissolution: Associations with concurrent and prospective distress and posttraumatic growth. Stress and Health, 33(5), 540-548. https://doi.org/10.1002/smi.2738

Feng, C., Wang, H., Lu, N., Chen, T., He, H., Lu, Y., \& Tu, X. M. (2014). Log-transformation and its implications for data analysis. Shanghai Archives of Psychiatry, 26(2), 105-109. 
Fischer, T., de Graaf, P., \& Kalmijn, M. (2005). Friendly and antagonistic contact between former spouses after divorce: Patterns and determinants. Journal of Family Issues, 26(8), 1131-1163. https://doi.org/10.1177/0192513X05275435

Fraley, R. C., \& Davis, K. E. (1997). Attachment formation and transfer in young adults' close friendships and romantic relationships. Personal Relationships, 4(2), 131-144.

Fujita, F., \& Diener, E. (2005). Life Satisfaction set point: Stability and change. Journal of Personality and Social Psychology, 88(1), 158-164. https://doi.org/10.1037/00223514.88.1.158

Hasselmo, K., Mehl, M. R., Tackman, A. M., Carey, A. L., Wertheimer, A. M., Stowe, R. P., \& Sbarra, D. A. (2018). Objectively measured social integration is associated with an immune risk phenotype following marital separation. Annals of Behavioral Medicine, 52(2), 130-145. https://doi.org/10.1093/abm/kax034

Hazan, C., \& Zeifman, D. (1994). Sex and the psychological tether. In K. Bartholomew \& D. Perlman (Eds.), Attachment processes in adulthood (pp. 151-178). London, England: Jessica Kingsley Publishers.

Lee, L. A., Sbarra, D. A., Mason, A. E., \& Law, R. W. (2011). Attachment anxiety, verbal immediacy, and blood pressure: Results from a laboratory-analogue study following marital separation. Personal Relationships, 18(2), 285-301. https://doi.org/10.1111/j.1475-6811.2011.01360.x

Lewandowski, G. W., \& Bizzoco, N. M. (2007). Addition through subtraction: Growth following the dissolution of a low quality relationship. The Journal of Positive Psychology, 2(1), 40-54. https://doi.org/10.1080/17439760601069234 
Manvelian, A., Bourassa, K. J., Lawrence, E., Mehl, M. R., \& Sbarra, D. A. (2018). With or without you? Loss of self following marital separation. Journal of Social and Clinical Psychology, 37(4), 297-324. https://doi.org/10.1521/jscp.2018.37.4.297

Masheter, C. (1997). Healthy and unhealthy friendship and hostility between ex-spouses. Journal of Marriage and Family, 59(2), 463-475. https://doi.org/10.2307/353483

Mason, A., Law, R., Bryan, A., Portley, R., \& Sbarra, D. (2012). Facing a breakup: Electromyographic responses moderate self-concept recovery following a romantic separation. Personal Relationships, 19(3), 551-568. https://doi.org/10.1111/j.14756811.2011.01378.x

Mason, A., \& Sbarra, D. (2012). Romantic separation, loss, and health: A review of moderators. Handbook of Health and Social Relationships. Washington, DC: American Psychological Association, 95-120.

Mason, A., Sbarra, D., Bryan, A., \& Lee, L. (2012). Staying connected when coming apart: The psychological correlates of contact and sex with an ex-partner. Journal of Social and Clinical Psychology, 31(5), 488-507. https://doi.org/10.1521/jscp.2012.31.5.488

Mehl, M. R. (2017). The Electronically Activated Recorder (EAR): A method for the naturalistic observation of daily social behavior. Current Directions in Psychological Science, 26(2), 184-190. https://doi.org/10.1177/0963721416680611

Mehl, M. R., Pennebaker, J. W., Crow, D. M., Dabbs, J., \& Price, J. H. (2001). The Electronically Activated Recorder (EAR): A device for sampling naturalistic daily activities and conversations. Behavior Research Methods, Instruments, \& Computers, 33(4), 517-523. https://doi.org/10.3758/BF03195410 
Mehl, M. R., Robbins, M. L., \& Deters, F. G. (2012). Naturalistic observation of health-relevant social processes: The electronically activated recorder methodology in psychosomatics. Psychosomatic Medicine, 74(4), 410-417. https://doi.org/10.1097/PSY.0b013e3182545470

Milek, A., Butler, E. A., Tackman, A. M., Kaplan, D. M., Raison, C. L., Sbarra, D. A., ... Mehl, M. R. (2018). "Eavesdropping on Happiness" revisited: A pooled, multisample replication of the association between life satisfaction and observed daily conversation quantity and quality. Psychological Science, 29(9), 1451-1462. https://doi.org/10.1177/0956797618774252

O’Hara, R. B., \& Kotze, D. J. (2010). Do not log-transform count data. Methods in Ecology and Evolution, 1(2), 118-122. https://doi.org/10.1111/j.2041-210X.2010.00021.x

Pinheiro, J., Bates, D., DebRoy, S., \& Sarkar, D. (2019). R Core Team (2019) nlme: Linear and nonlinear mixed effects models. R package version 3.1-141. Available at $h$ Ttp://CRAN. R-Project. Org/Package= Nlme.

Prigerson, H. G., Maciejewski, P. K., Reynolds III, C. F., Bierhals, A. J., Newsom, J. T., Fasiczka, A., ... Miller, M. (1995). Inventory of Complicated Grief: A scale to measure maladaptive symptoms of loss. Psychiatry Research, 59(1-2), 65-79.

Rhoades, G. K., Kamp Dush, C. M., Atkins, D. C., Stanley, S. M., \& Markman, H. J. (2011). Breaking up is hard to do: The impact of unmarried relationship dissolution on mental health and life satisfaction. Journal of Family Psychology, 25(3), 366-374. https://doi.org/10.1037/a0023627

Robbins, M. L., Focella, E. S., Kasle, S., López, A. M., Weihs, K. L., \& Mehl, M. R. (2011). Brief report: Naturalistically observed swearing, emotional support and depressive 
symptoms in women coping with illness. Health Psychology, 30(6), 789-792. https://doi.org/10.1037/a0023431

Sbarra, D. A., Boals, A., Mason, A. E., Larson, G. M., \& Mehl, M. R. (2013). Expressive writing can impede emotional recovery following marital separation. Clinical Psychological Science, 1(2), 120-134.

Sbarra, D. A., \& Emery, R. E. (2005a). Coparenting conflict, nonacceptance, and depression among divorced adults: Results from a 12-year follow-up study of child custody mediation using multiple imputation. The American Journal of Orthopsychiatry, 75(1), 63-75. https://doi.org/10.1037/0002-9432.75.1.63

Sbarra, D. A., \& Emery, R. E. (2005b). The emotional sequelae of nonmarital relationship dissolution: Analysis of change and intraindividual variability over time. Personal Relationships, 12(2), 213-232. https://doi.org/10.1111/j.1350-4126.2005.00112.x

Sbarra, D. A., Emery, R. E., Beam, C. R., \& Ocker, B. L. (2014). Marital dissolution and major depression in midlife: A propensity score analysis. Clinical Psychological Science, 2(3), 249-257. https://doi.org/10.1177/2167702613498727

Shrout, P. E. (1998). Measurement reliability and agreement in psychiatry. Statistical Methods in Medical Research, 7(3), 301-317. https://doi.org/10.1177/096228029800700306

Slatcher, R. B., \& Robles, T. F. (2012). Brief report: Preschoolers' everyday conflict at home and diurnal cortisol patterns. Health Psychology, 31(6), 834-838. https://doi.org/10.1037/a0026774

Tackman, A. M., Sbarra, D. A., Carey, A. L., Donnellan, M. B., Horn, A. B., Holtzman, N. S., ... Mehl, M. R. (2019). Depression, negative emotionality, and self-referential language: A 
multi-lab, multi-measure, and multi-language-task research synthesis. Journal of Personality and Social Psychology, 116(5), 817-834.

Tobin, E. T., Kane, H. S., Saleh, D. J., Wildman, D. E., Breen, E. C., Secord, E., \& Slatcher, R. B. (2015). Asthma-related immune responses in youth with asthma: Associations with maternal responsiveness and expressions of positive and negative affect in daily life. Psychosomatic Medicine, 77(8), 892-902. https://doi.org/10.1097/PSY.0000000000000236

Vazire, S., \& Mehl, M. R. (2008). Knowing me, knowing you: The accuracy and unique predictive validity of self-ratings and other-ratings of daily behavior. Journal of Personality and Social Psychology, 95(5), 1202-1216. https://doi.org/10.1037/a0013314

Verhallen, A. M., Renken, R. J., Marsman, J.-B. C., \& Horst, G. J. ter. (2019). Romantic relationship breakup: An experimental model to study effects of stress on depression (like) symptoms. PLOS ONE, 14(5), e0217320. https://doi.org/10.1371/journal.pone.0217320

Wang, H., \& Amato, P. R. (2000). Predictors of divorce adjustment: Stressors, resources, and definitions. Journal of Marriage and Family, 62(3), 655-668.

Wei, M., Russell, D. W., Mallinckrodt, B., \& Vogel, D. L. (2007). The Experiences in Close Relationship Scale (ECR)-short form: Reliability, validity, and factor structure. Journal of Personality Assessment, 88(2), 187-204.

Weiss, D. S. (2007). The impact of event scale: Revised. In J. Wilson \& C. Tang (Eds.), Crosscultural assessment of psychological trauma and PTSD (pp. 219-238). New York, NY: Springer. 
Weissman, M. M., Bland, R. C., Canino, G. J., Greenwald, S., Hwu, H.-G., Joyce, P. R., ... Lepine, J.-P. (1999). Prevalence of suicide ideation and suicide attempts in nine countries. Psychological Medicine, 29(1), 9-17. 
Table 1.

Descriptive Statistics and Bivariate Correlations of Study Variables

\begin{tabular}{|c|c|c|c|c|c|c|c|c|c|c|c|c|c|c|c|}
\hline & 1 & 2 & 3 & 4 & 5 & 6 & 7 & 8 & 9 & 10 & 11 & 12 & 13 & 14 & 15 \\
\hline 1. WithEx T1 & 1 & & & & & & & & & & & & & & \\
\hline 2. WithEx T3 & 0.77 & 1 & & & & & & & & & & & & & \\
\hline 3. WithEx T5 & 0.80 & 0.90 & 1 & & & & & & & & & & & & \\
\hline 4. $\quad$ SRPD T1 & 0.23 & 0.19 & 0.12 & 1 & & & & & & & & & & & \\
\hline 5. SRPD T3 & 0.22 & 0.06 & 0.07 & 0.74 & 1 & & & & & & & & & & \\
\hline 6. SRPD T5 & 0.16 & 0.16 & 0.11 & 0.71 & 0.75 & 1 & & & & & & & & & \\
\hline $\begin{array}{ll}\text { 7. } & \text { Perceived } \\
& \text { Responsibility }\end{array}$ & -0.03 & -0.05 & -0.13 & 0.23 & 0.15 & 0.14 & 1 & & & & & & & & \\
\hline 8. Age (years) & -0.04 & -0.08 & -0.02 & 0.00 & 0.06 & 0.10 & -0.10 & 1 & & & & & & & \\
\hline 9. Sex & -0.01 & -0.01 & -0.07 & 0.21 & 0.18 & 0.25 & 0.06 & 0.11 & 1 & & & & & & \\
\hline $\begin{array}{l}\text { 10. Relationship Length } \\
\text { (months) }\end{array}$ & -0.16 & -0.17 & -0.17 & -0.18 & -0.13 & -0.02 & -0.06 & 0.62 & 0.11 & 1 & & & & & \\
\hline $\begin{array}{l}\text { 11. Separation Length } \\
\text { (months) }\end{array}$ & -0.12 & -0.16 & -0.16 & 0.00 & 0.08 & 0.15 & 0.16 & 0.00 & 0.00 & 0.05 & 1 & & & & \\
\hline 12. Attachment Avoidance & 0.17 & 0.09 & 0.2 & 0.15 & 0.25 & 0.17 & -0.22 & 0.09 & 0.10 & -0.04 & 0.05 & 1 & & & \\
\hline 13. Attachment Anxiety & 0.15 & 0.15 & 0.16 & 0.40 & 0.38 & 0.30 & 0.09 & -0.21 & 0.09 & -0.21 & 0.10 & 0.04 & 1 & & \\
\hline 14. Lingering Attachment & 0.13 & 0.20 & 0.16 & 0.27 & 0.16 & 0.21 & -0.10 & 0.08 & 0.24 & -0.06 & -0.08 & -0.02 & 0.20 & 1 & \\
\hline 15. Children & 0.18 & 0.03 & 0.04 & -0.14 & -0.08 & -0.01 & 0.11 & 0.27 & 0.04 & 0.56 & -0.08 & 0.18 & -0.05 & -0.15 & 1 \\
\hline Mean & 1.17 & 1.58 & 1.70 & 30.42 & 24.15 & 20.69 & 2.77 & 43.84 & - & 187.49 & 3.76 & 2.86 & 3.84 & 2.74 & - \\
\hline SD & 3.46 & 6.13 & 5.93 & 15.91 & 15.62 & 15.40 & 0.97 & 10.52 & - & 112.98 & 2.17 & 1.07 & 1.23 & 1.51 & - \\
\hline
\end{tabular}

Note: SD = standard deviation; SRPD = separation-related psychological distress; WithEx T1-T3 summary statistics represent the percentage of sound files in which the participant was with their ex-partner. 
Table 2.

Unstandardized Regression Coefficients from Models Predicting Concurrent SRPD

\begin{tabular}{|c|c|c|c|}
\hline Model 1: Baseline Change Model & $b$ & $\mathrm{SE}$ & $95 \% \mathrm{CI}$ \\
\hline Time & $-4.57 * *$ & 0.55 & $-5.64,-3.50$ \\
\hline Between-Person (grand-mean centered) WithEx & 46.32 & 25.99 & $-4.84,97.47$ \\
\hline Within-Person (person-mean centered) WithEx & -31.72 & 19.89 & $-70.70,7.26$ \\
\hline Model 2: Baseline Change Model with All Covariates & $b$ & $\mathrm{SE}$ & $95 \% \mathrm{CI}$ \\
\hline Time & $-4.55 * *$ & 0.61 & $-5.73,-3.36$ \\
\hline Between-Person (grand-mean centered) WithEx & 29.14 & 26.55 & $-22.50,80.78$ \\
\hline Within-Person (person-mean centered) WithEx & -12.08 & 23.20 & $-56.97,32.81$ \\
\hline Age & $0.28 *$ & 0.13 & $0.03,0.54$ \\
\hline Sex & 3.68 & 2.48 & $-1.14,8.50$ \\
\hline Separation Length & 0.10 & 0.52 & $-0.92,1.12$ \\
\hline Relationship Length & $-0.03 *$ & 0.01 & $-0.05,-0.001$ \\
\hline Perceived Responsibility for Separation & $3.53 * *$ & 1.18 & $1.23,5.83$ \\
\hline Attachment Anxiety & $3.99 * *$ & 0.94 & $2.15,5.82$ \\
\hline Attachment Avoidance & $2.66^{*}$ & 1.08 & $0.60,4.81$ \\
\hline Lingering Attachment & 1.23 & 0.77 & $-0.26,2.72$ \\
\hline $\begin{array}{l}\text { Model 3: Moderated In-person contact (Attachment } \\
\text { Anxiety) }\end{array}$ & $b$ & $\mathrm{SE}$ & $95 \% \mathrm{CI}$ \\
\hline Time & $-4.62 * *$ & 0.58 & $-5.76,-3.49$ \\
\hline Between-Person (grand-mean centered) WithEx & 22.41 & 23.82 & $-24.27,69.08$ \\
\hline Within-Person (person-mean centered) WithEx & 107.59 & 87.75 & $-63.57,278.75$ \\
\hline Attachment Anxiety & $4.31 * *$ & 0.95 & $2.45,6.17$ \\
\hline Attachment Avoidance & $2.41 *$ & 1.10 & $0.26,4.55$ \\
\hline Within-Person WithEx * Attachment Anxiety & -32.35 & 19.37 & $-70.13,5.43$ \\
\hline $\begin{array}{l}\text { Model 4: Moderated In-person contact (Lingering } \\
\text { Attachment) }\end{array}$ & $b$ & $\mathrm{SE}$ & $95 \% \mathrm{CI}$ \\
\hline Time & $-4.59 * *$ & 0.55 & $-5.67,-3.52$ \\
\hline Between-Person (grand-mean centered) WithEx & 35.74 & 25.80 & $-14.88,86.37$ \\
\hline Within-Person (person-mean centered) WithEx & $-138.86^{*}$ & 57.15 & $-250.51,-27.21$ \\
\hline Lingering Attachment & $2.04 *$ & 0.84 & $0.39,3.70$ \\
\hline Within-Person WithEx * Lingering Attachment & $31.49 *$ & 15.70 & $0.82,62.16$ \\
\hline
\end{tabular}


Table 3.

Unstandardized Regression Coefficients from Models Predicting Lagged SRPD

\begin{tabular}{lccc}
\hline Model 5: Lagged Effect Model & $b$ & SE & $95 \%$ CI \\
\hline Time & $-3.48^{* *}$ & 1.04 & $-5.52,-1.45$ \\
Between-Person (grand-mean centered) WithEx & 37.98 & 33.67 & $-27.94,103.89$ \\
Within-Person (person-mean centered) WithEx & 70.76 & 71.88 & $-70.26,211.78$ \\
Within-Person WithEx Lag-1 & $123.15^{* *}$ & 32.32 & $59.74,186.57$ \\
\hline Model 6: Lagged Interaction Effect Model with CVs & $b$ & SE & $95 \%$ CI \\
\hline Time & $-4.22^{* *}$ & 1.19 & $-6.48,1.96$ \\
Between-Person (grand-mean centered) WithEx & 29.35 & 37.13 & $-41.32,100.02$ \\
Within-Person (person-mean centered) WithEx & 49.70 & 63.85 & $-72.04,171.44$ \\
Within-Person WithEx Lag-1 & $226.14^{* *}$ & 61.32 & $109.24,343.05$ \\
Age & 0.29 & 0.16 & $-0.02,0.59$ \\
Sex & 2.49 & 2.99 & $-3.20,8.18$ \\
Separation Length & 0.59 & 0.62 & $-0.59,1.77$ \\
Relationship Length & -0.02 & 0.02 & $-0.05,0.02$ \\
Perceived Responsibility for Separation & $3.22^{*}$ & 1.41 & $0.54,5.91$ \\
Attachment Anxiety & $3.27^{*}$ & 1.08 & $1.20,5.33$ \\
Attachment Avoidance & $3.37^{*}$ & 1.28 & $0.94,5.81$ \\
Lingering Attachment & 0.40 & 0.93 & $-1.38,2.17$ \\
Children & -4.54 & 3.50 & $-11.19,2.11$ \\
Within-Person WithEx Lag-1*Children & $-223.41^{*}$ & 74.54 & $-223.41,-81.29$ \\
\hline Nit SRPD sop
\end{tabular}

Note: SRPD = separation-related psychological distress; $b=$ unstandardized regression coefficient; $S E=$ standard error; $\mathrm{CI}=$ confidence interval; $\mathrm{CV}=$ covariate. Significance levels: * $\mathrm{p}<.05, * * \mathrm{p}<.001$. 


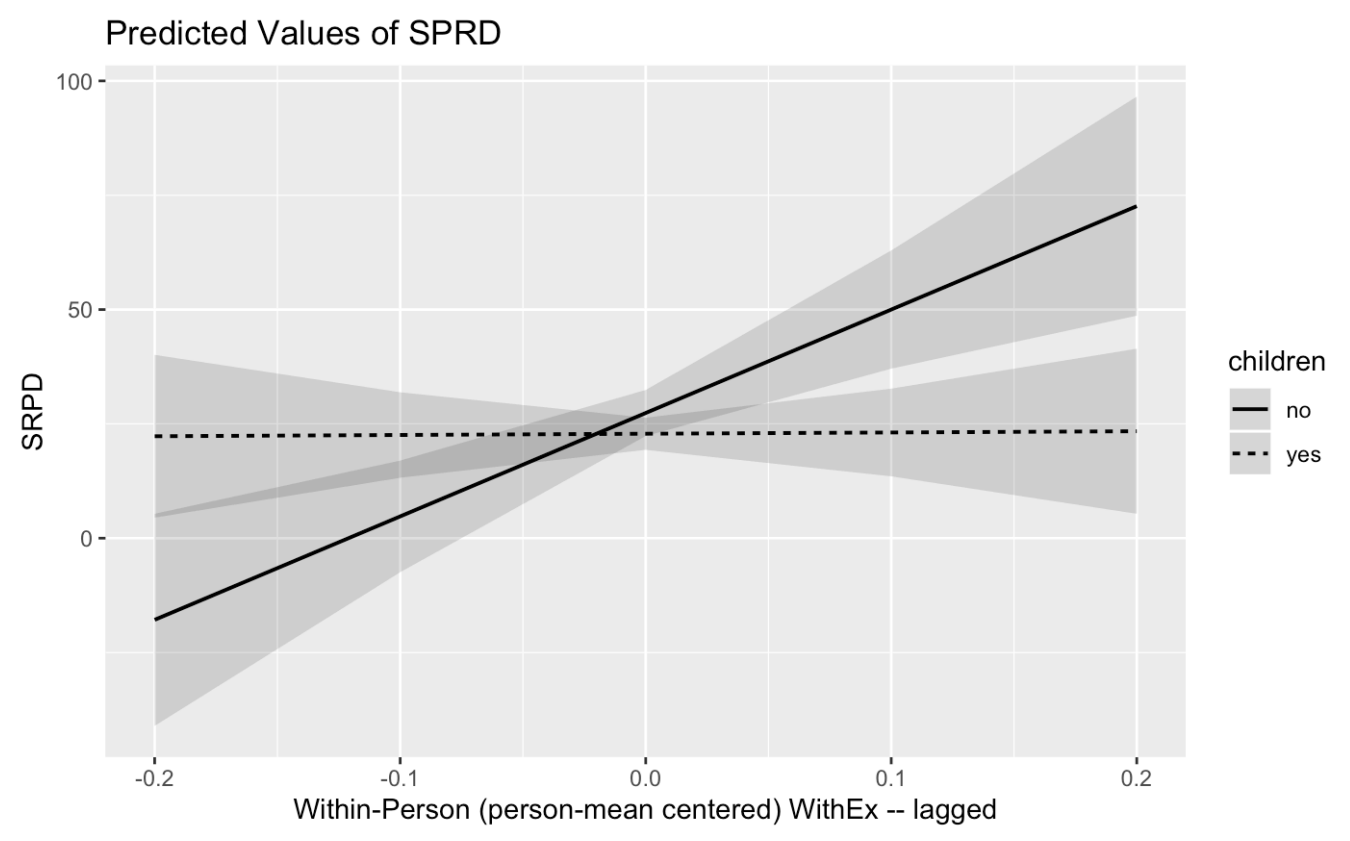

Figure 1. Predicted association between Within-Person WithEx (lagged) and SRPD as a function of whether or not an individual has children with their ex-partner; illustration of Model 6:

Lagged Interaction Effect Model with All Covariates.

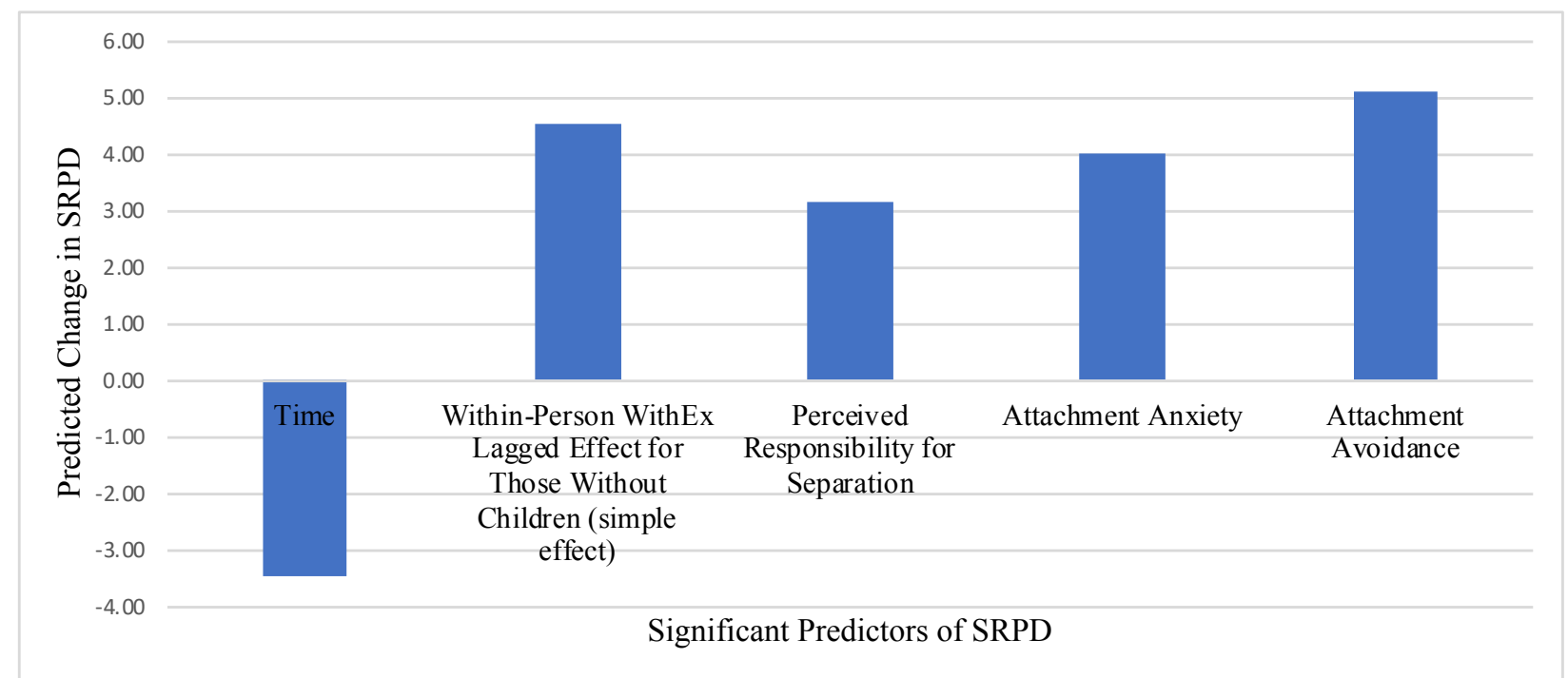

Figure 2. Relative magnitude of change in SRPD associated with 1 SD increase in each of the five significant predictors of SRPD in Model 6: Lagged Interaction Effect Model with All Covariates. Note that the effect of Within-Person WithEx Lagged is the simple effect for those without children. 